\title{
Globe
}

Revue internationale d'études québécoises

\section{Jose del Pozo, Les Chiliens au Québec. Immigrants et réfugiés, de 1955 à nos jours, Montréal, Boréal, 2009}

\section{Victor Armony}

Volume 13, numéro 1, 2010

Culture et relations internationales

URI : https://id.erudit.org/iderudit/044648ar

DOI : https://doi.org/10.7202/044648ar

Aller au sommaire du numéro

Éditeur(s)

Globe, Revue internationale d'études québécoises

ISSN

1481-5869 (imprimé)

1923-8231 (numérique)

Découvrir la revue

Citer ce compte rendu

Armony, V. (2010). Compte rendu de [Jose del Pozo, Les Chiliens au Québec. Immigrants et réfugiés, de 1955 à nos jours, Montréal, Boréal, 2009]. Globe, 13(1), 179-182. https://doi.org/10.7202/044648ar d'utilisation que vous pouvez consulter en ligne.

https://apropos.erudit.org/fr/usagers/politique-dutilisation/ 
nauté juive de Montréal. À priori, une telle position, qui peut influencer l'interprétation des sources historiques, est généralement évitée par les historiens. Un autre indicateur de la nature parfois démagogique de ce texte est l'usage excessif du point d'exclamation, une pratique rare dans les ouvrages plus formels.

Quoique Fabled City soit un recueil fascinant d'anecdotes, de faits et d'opinions, sa valeur historique n'est pas constante. Si King offre une bibliographie, l'absence de références dans le corps du texte rend néanmoins difficile toute vérification de renseignements. En revanche, la présence de nombreuses photographies et documents donne vie aux récits, et contribue à faire de Fabled City une importante introduction à la culture populaire des Juifs montréalais.

Steven Lapidus Université Concordia

\section{Jose del Pozo \\ Les Chiliens au Québec. Immigrants et réfugiés, de 1955 à nos jours, Montréal, Boréal, 2009.}

La communauté chilienne occupe une place remarquable dans la société québécoise, non pas par sa taille ou son influence, mais pour des raisons historiques et sociologiques particulières qui, d'ailleurs, permettent d'éclairer l'évolution des rapports entre la majorité et les minorités. Il va de soi que toute étude de cas d'un groupe issu de l'immigration est pertinente et le cas des Chiliens, même s'il est relativement mieux connu que d'autres, méritait bien qu'un ouvrage entier lui soit consacré. Outre les spécialistes, bien des lecteurs québécois tireront profit d'un livre qui retrace avec minutie la trajectoire de cette fascinante communauté, traversée par des enjeux politiques, économiques et culturels. José del Pozo, lui-même d'origine chilienne, a vécu cette trajectoire de l'intérieur et, bien évidemment, en possède une connaissance intime. Ses indubitables qualités d'historien donnent au travail la rigueur nécessaire, sans laquelle ce type de portrait aurait pu sombrer dans la description sèche, sans profondeur analytique. En fait, dans le but de maintenir une distance convenable vis-à-vis de son objet, l'auteur a constitué un ample échantillon de répondants et s'est appuyé sur 
une approche méthodologique soigneusement exposée en annexe. Bien que l'on puisse rester quelque peu sceptique à l'égard de la représentativité des répondants (choisis selon le principe de la "boule de neige", mais relevant, dans la pratique, de ce que les statisticiens appellent "un échantillonnage de commodité»), il n'en demeure pas moins qu'il s'agit d'une solide étude qualitative.

La première partie du livre présente, de manière chronologique, la «spécificité du flux migratoire de chaque période» (p. 12), en tenant compte des contextes chilien, québécois et canadien. Les chapitres couvrent les périodes de l'" exode des cerveaux» (les décennies 1950 et 1960), des "années de plomb» (1973-1978), du "refuge économique» (1979-1989) et de la «normalisation" (depuis 1990). On peut supposer que le lecteur moyen trouvera, dans les pages consacrées à l'ère Pinochet, les repères qui lui sont les plus familiers. En effet, c'est autour des demandeurs d'asile politique que la vie communautaire chilienne s'est surtout bâtie au Québec, mais c'est aussi par les liens de solidarité - et idéologiques - qui se sont tissés durant ces années que le Chili, comme puissant symbole d'espoir et de résistance en Amérique latine, a acquis une dimension nouvelle dans l'imaginaire québécois (alors marqué par le rêve de la «libération nationale»). Cependant, l'auteur évite le romantisme: l'histoire des Chiliens au Québec n'est pas peuplée que de victimes de l'oppression, de résistants ou de patriotes forcés à l'exile. Nombre de ceux qui ont quitté leur pays ont été «motivés en partie par la présence de la dictature mais aussi par des raisons plus diversifiées" (p. 68), parmi lesquelles les raisons économiques sont souvent - quoique pas toujours - déterminantes. C’est pourquoi, dans les mots de José del Pozo, "l'expérience migratoire des Chiliens au Québec a un caractère plus complexe qu'il n'y paraît à première vue " (p. 327) et donnera lieu à un phénomène des plus intrigants: «alors que le Chili commençait à sortir de la dictature, le nombre de candidats au statut de réfugié au Québec a augmenté» (p. 95). Bien que l'auteur observe ce type de paradoxe sans véritablement l'explorer ou le problématiser (démontrant une retenue admirable en termes de neutralité scientifique, mais quelque peu décevante sous l'angle de la discussion intellectuelle), on ne peut que saluer sa volonté d'échapper aux clichés et aux réductionnismes.

La deuxième partie du livre constitue le noyau sociologique de l'étude: l'auteur se penche sur «la vie des Chiliens au Québec»: l'insertion dans le marché du travail, la participation civique et politique, la vie associative et la production culturelle. S'appuyant sur une masse de renseignements, José del Pozo décrit la communauté et propose un bilan, 
somme toute, assez positif. La situation économique et professionnelle des Chiliens au Québec "n'est pas rose", mais l'avenir se montre "plus prometteur" (p. 176), tandis que l'intégration civique constitue une "expérience réussie" (p. 177). Le mouvement associatif de cette communauté "s'est manifesté à travers une vaste panoplie d'activités et d'intérêts» (p. 236) et sa contribution à l'essor artistique du Québec est considérable, les écrivains et peintres d'origine chilienne ayant «investi un vaste champ d'activités» (p. 264). Malgré le strict réalisme dont l'auteur fait preuve - qui l'amène à moduler ses interprétations et à signaler autant les succès que les échecs chez ce groupe d'immigrants et leurs descendants -, le lecteur peut se demander si la vision d'ensemble n'est pas néanmoins biaisée par l'échantillonnage (ce qui, par ailleurs, rend l'utilisation de pourcentages pour, par exemple, mesurer l'appui des Chiliens à la souveraineté, hautement contestable [page 196]). Les personnes interviewées (notamment dans le groupe qui a été la principale source des témoignages oraux) ne sont-elles pas généralement - presque par définition - bien intégrées, appartenant à la classe moyenne, parfois même des têtes d'affiche de la communauté ou de leur domaine d'activité? Elles constituent sans doute le cœur et le visage de la minorité chilienne québécoise, mais il y a lieu de s'interroger sur les hommes et les femmes qui ont été moins au centre de la vie associative ou dont le parcours biographique a été moins remarqué.

Les deux derniers chapitres offrent une perspective différente, davantage centrée sur le monde intérieur, sur la subjectivité des immigrants, que sur leurs actions. L'auteur s'attarde sur les sentiments (peur, rage, soulagement) des individus, sur les rapports au sein des couples et avec les enfants, sur la "question identitaire", sur les déchirements (entre le pays d'accueil et le pays d'origine) et les désillusions (chez ceux qui sont retournés au Chili). Ces pages sont extrêmement stimulantes et complètent le tableau d'une communauté riche en nuances. On peut toutefois regretter que l'auteur n'ait pas profité de ce cadre pour avancer quelques idées sur les particularités du trait d'union chilien-québécois. Autrement dit, un livre sur les Chiliens au Québec devrait idéalement distinguer (et comparer) les Chiliens des autres immigrants d'origine latino-américaine qui se sont établis au Québec, comme il devrait situer le Québec par rapport à d'autres sociétés qui ont accueilli des Chiliens. Ce n'est pas le projet auquel s'est attaqué José del Pozo et son choix est tout à fait légitime. Mais l'inclusion de quelques éléments allant dans ce sens aurait enrichi significativement sa démarche. En dépit de ces limites, il ne fait pas de doute que cet ouvrage représente une contribution majeure à l'histoire de l'immigration chilienne et sa lecture est 
indispensable à ceux qui s'intéressent au devenir de la société québécoise à travers le prisme des divers groupes qui la composent.

Victor Armony

Université du Québec à Montréal

\section{Gilles Marcotte}

La littérature est inutile,

Montréal, Éditions du Boréal, 2009.

Sans aller jusqu'à dire qu'il faut trembler devant l'homme et son œuvre, on n'aborde pas un ouvrage de Gilles Marcotte comme on lit la première ou la dernière livraison du printemps. Non seulement l'auteur de La littérature est inutile a une vue imprenable et unique sur la littérature canadienne-française puis québécoise, mais il l'a aussi et surtout alimentée, informée à force d'essais critiques et de comptes rendus qui remontent jusqu'aux années 1940. À l'aune de la bibliographie de Gilles Marcotte, ma décennie en littérature n'est pas un gage de témérité: elle engendre bien plutôt l'impression d'avoir manqué plusieurs épisodes que lui, le critique et universitaire, a vus, revus et mieux compris que quiconque. Il me reste un peu d'insouciance.

Il ne faut pas se laisser berner par le titre, inutilement provocateur. Il y a d'abord l'intention, qui arrive, comme c'est souvent le cas dans la collection "Papiers collés», a posteriori: "J'ai voulu [...] que les œuvres, les écrivains que je présente ici le soient pour eux-mêmes, en eux-mêmes, sans être conscrits par une sorte de développement collectif.» (p.11) Des prémices kundériennes - «[l']œuvre authentiquement littéraire est celle qui rend le jugement impossible»; elle apprend à lire "la complexité, l'infinie complexité de l'aventure humaine» (p. 9) - donnent à penser que l'auteur se détourne quelque peu de son titre de héraut de la sociocritique, conféré par les tenants de «l'École de Montréal». Même si Marcotte est prudent - «la réunion d'œuvres parues dans le même espace géographique ne peut que suggérer des perspectives historiques, des relations entre texte et société»-, il précise que son "propos est différent" (p. 11). Le résultat, c'est-à-dire cette collection d'essais épars consacrés à la littérature québécoise qui vont de 1987 à 2008 , ne se confond pas tout à fait avec cette volonté «initiale». À lire $L a$ littérature est inutile, on pense encore et toujours à Littérature et circonstances 